\title{
Cutaneous Aspergilosis Caused by Aspergillus Flavus: A Case Report
}

\author{
Maria Ulfa Sheilaadji, Indropo Agusni, Linda Astari, Sylvia Anggraeni, Yuri Widia, Evy \\ Ervianti \\ Faculty of Medicine, Universitas Airlangga, Dr. Soetomo General Academic Teaching Hospital, \\ Surabaya, Indonesia
}

\begin{abstract}
Background: Cutaneous aspergillosis occurs relatively less frequent and therefore remains poorly characterized. Cutaneous aspergillosis can be as primary or secondary infection. Primary cutaneous aspergillosis usually involves sites of skin injury, intravenous catheter, traumatic inoculation, and associated with occlusive dressings. Secondary lesions result from contiguous extension from infected underlying structures or from widespread blood-borne seeding of the skin. Purpose: To know the skin manifestation, efflorence, examination and therapy of cutaneous aspergillosis. Case: A man complaint itchy redness macule and pimples on the right arm since 2 weeks. Initially just felt a little then expands. Patients with post operative brachial injury and uses a cast during one month. On examination there are erythematous macule unsharply marginated with papules. Potassium hydroxide examination, shows conidiophores, dichotomously branching and septate hyphae appropriate description with Aspergillosis Sp. Cultures found grow granular colonies, flat often with radial grooves, yellow at first but quickly becoming bright to dark yellow-green with age, For the identification microscope from the culture specimen there was conidia, phialde, conidiophore and vesicle that suitable with Aspergillus flavus. Patients received itraconazole 2 x $200 \mathrm{mg}$ for 6 weeks and obtained satisfactory results. Discussion: Healthy hosts can develop cutaneous aspergillosis in surgical wounds, by traumatic inoculation, at sites associated with occlusive dressings. In some instances, a presumptive diagnosis of primary cutaneous aspergillosis can be made immediately by examining a potassium hydroxide preparation and culture. Conclusion: Diagnose of cutaneous aspergillosis can establish by potassium hydroxide and culture examination, therapy with itraconazole $2 \mathrm{x} 200 \mathrm{mg}$ give satisfactory results.
\end{abstract}

Keywords: Cutaneous aspergillosis, Aspergillus flavus, potassium hydroxide, culture.

Correspondence: Evy Ervianti, Department of Dermatology and Venereology, Faculty of Medicine Universitas Airlangga, Dr. Soetomo General Academic Teaching Hospital, Jl.Mayjend Prof Dr. Moestopo No.6-8 Surabaya 60131, Indonesia, phone: (031) 5501609, email: evy_if@yahoo.co.id

\section{BACKGROUND}

Cutaneous aspergillosis is a rare disease and may occur as either primary or secondary infection. In primary cutaneous aspergillosis, the lesion occurs as a result of direct inoculation of Aspergillus spores at the site of injury following intravenous catheter, trauma, occlusive dressings and tapes, burns or surgery. In secondary cutaneous aspergillosis, the lesions occur due to haematogenous dissemination from a primary focus such as the lungs or to contiguous spread to the skin from underlying infected structures. ${ }^{1}$ Although over 300 species of aspergillus exist, over $90 \%$ of human infection is by Aspergillus fumigatus, typically causing systemic infection involving the lungs, blood, and sinuses; primary skin infection is usually caused by Aspergillus flavus, terreus, niger and $u t u s .{ }^{2}$ Cutaneous manifestations are non specific and can be presented with erythematoviolaceous patches that have a central necrotic ulcer, subcutaneous abscesses and vegetative papules and patches. ${ }^{3}$
We report a case of cutaneous aspergillosis in a 22 years-old male patient, redness macule and pimples on right arm, who got clinical improvement by itraconazole. This report discusses about the clinical presentation, diagnosis, and treatment.

\section{CASE REPORT}

Twenty two years-old male patient presents with redness macule and pimples on his right arm since 2 weeks, felt itchy in that area. Patient that consulted from orthopedic outpatient clinic with post operation brachial injury, patient suffer from traffic accident fall from the motorcycle two months ago and underwent operation one month ago. After surgery the patient using a cast on his right arm during the first month and never released during that first month. Patients are aware of the complaint since two weeks ago when the patient control to orthopedic outpatient clinic to replace the cast with bandage and found that there are itchy redness macule and papule, become wider in 2 weeks. 
Patient not routinely takes a bath and cleanse the body during use cast and bandage. Patient are unmarried man and never had sexual intercourse. Patients never treat complaints with oral medication or topical drug. The patient didn't complain about the same redness macule and pimples on the other body parts and no complaints of fever.

Physical examination showing no abnormalities, blood presure: 110/70 $\mathrm{mmHg}$, heart rate: $88 \mathrm{x} /$ minute, respiratory rate: $16 \mathrm{x} /$ minute, and temperature : $36,2^{\circ} \mathrm{C}$ General condition is good with the glasgow coma scale is 4-5-6. From head and neck, thorax, abdomen, and extremities all within normal limit. The body mass index is 23 and categorized as normal. From dermatological examination there are erythematous macule unsharply marginated with papule and no pustule.
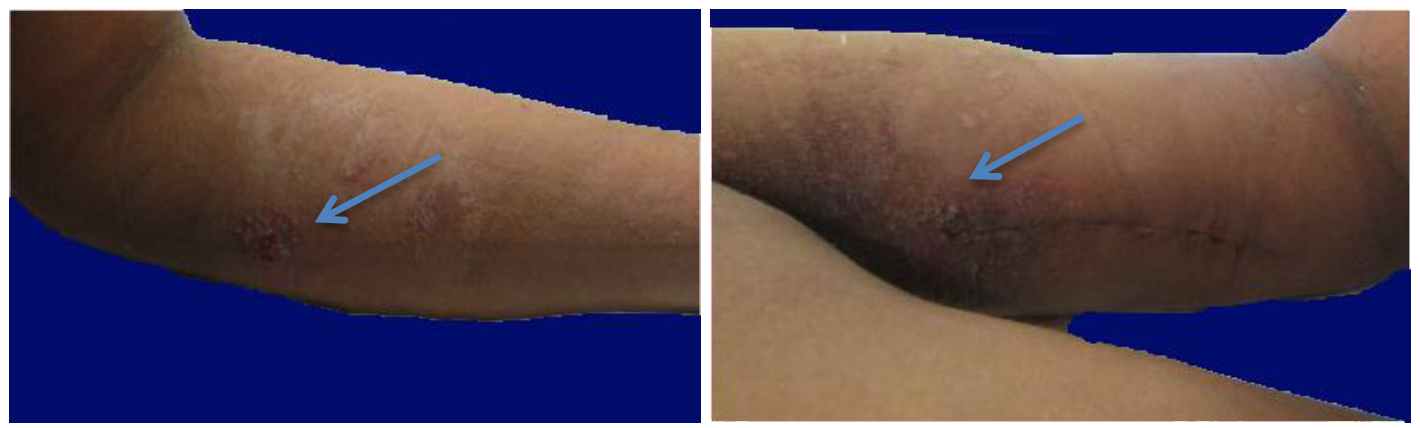

Figure 1. Multiple papules on erythematous macule in brachialis area felt itchy on it that show by the arrow.

From the anamnesis and physical ecxamination firsly the diagnosed was candidiasis cutis with some differensial diagnose like dermatitis, tinea corporis and erythema annulare. The potassium hydroxide and culture were performed to make the right diagnosis. Potassium hydroxide examination shows conidiophores, dichotomously branching and septate hyphae that appropriate description with Aspergillosis $s p$. Based on the history, clinical manifestation, and examination, the patient was diagnosed by cutaneous aspergillosis. To determine the specific species of aspergillosis we need do the culture examination of the skin. In addition to knowing the risk factors in patients we check HIV rapid test examination and to determine the involvement of fungal in circulation, we check blood cultures examination.
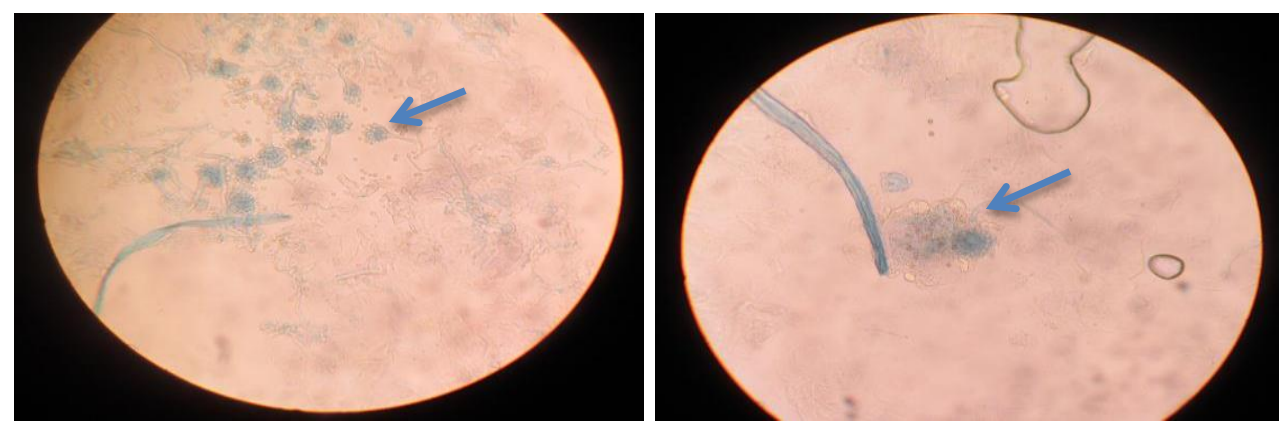

Figure 2. Potassium Hydroxide examination, the arrow shows conidiophores, dichotomously branching and septate hyphae.

The result of culture that grow in Sabouraud Dextrose Agar (SDA) media that grow in day $12^{\text {nd }}$ shows the fungal colonies are granular, flat often with radial grooves, yellow at first but quickly becoming bright to dark yellow-green according age. After that, the microscopic examination was performed a picture that appropriate with Aspergillus flavus, conidial head are typically radiate, later splitting to form loose column, biseriate but having some head with phialides borne directly on the vesicle (unisariate), conidiophore stripes are hyaline and coarsely roughed often more noticeable near the vesicle. Blood examination result shows there are no increasing leukocytes, eosinophil, and the other with in normal limit. The rapid HIV screening test result is negative (non reactive), blood culture results showed there was no growth of fungi. In this case the biopsy should be performed to support the results of culture in determining the species but the patient refused to do the biopsy because still complained of pain in the area that will do the biopsy. 

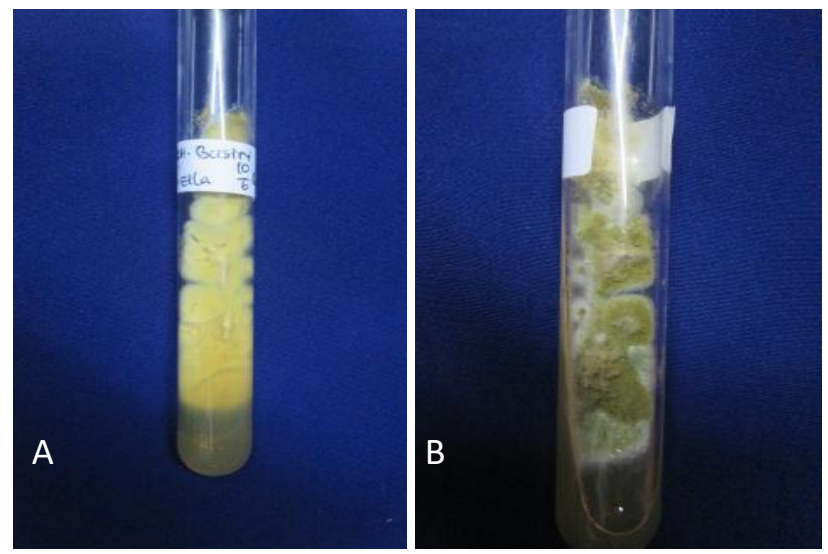

Figure 3. The macroscopic culture that grow in 12nd days. A. Back. B. Front, shows the fungal colonies are granular with radial grooves and with dark yellow-green color.
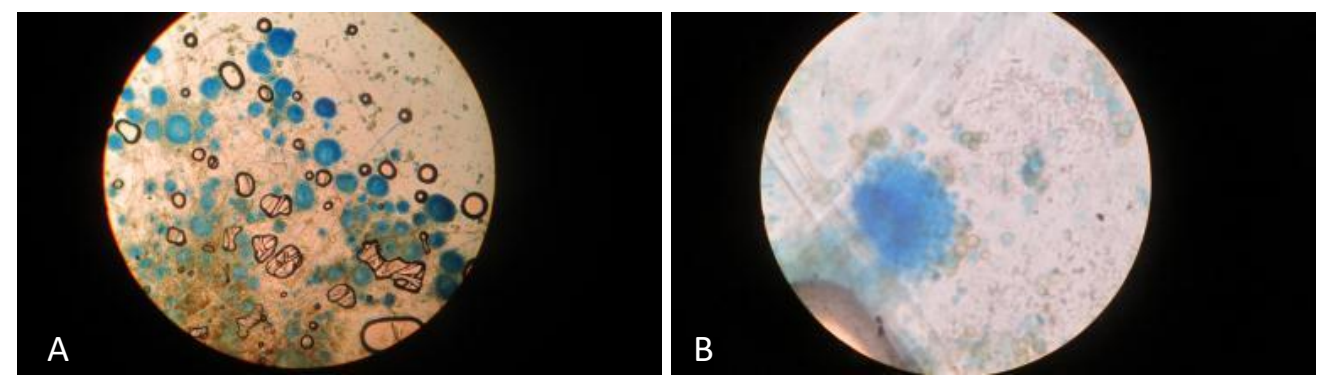

Figure 4. The microscopic culture. A. 100x magnification. B. 400x magnification. The arrow show conidia head and phialdes

Based on the history, clinical manifestation, and all the examination, the patient was diagnosed with cutaneous aspergillosis that caused by Aspergillus flavus. From that diagnosis, the patients received therapy itraconazol 2 x $200 \mathrm{mg}$ during the first 2 weeks and then control to outpatient clinic, at the time of control patients are routinely checked potassium hydroxide to assess the response of therapy. In addition patients were also given education in order to maintain the hygiene of the body, especially the area covering the lesion.
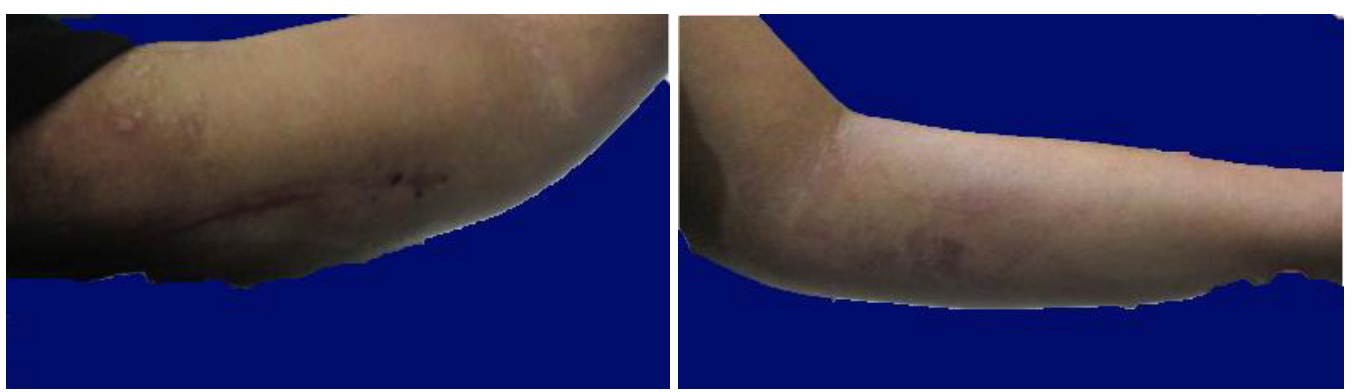

Figure 5. Improvement after 4 weeks treatment, there is no more itchy erythematous macule and papul.

Patient received itraconazole therapy for 4 weeks and the results obtained potassium hydroxide that is already out of the picture that fit the description Aspergillus Sp., but only found a lot of hyphae, then treatment continued for 2 weeks and there is an excellent therapeutic response.

\section{DISCUSSION}

Male, 22 years old patient suffered from cutaneous aspergillosis with post operation brachial injury that using cast for a month. This is consistent with literature that the risk factor primer cutaneous aspergillosis uses dressing. Cutaneous aspergillosis occurs relatively less frequently and therefore remains 
poorly characterized. Previous reports have described cutaneous aspergillosis can be either primary or secondary, which results from disseminated aspergillosis. Primary cutaneous lesions result from direct inoculation of the aspergillus species from trauma, especially in patients on catheter, trauma from an arm board, burns, contaminated dressings, and cases have been reported in the neonatal Intensive Care Units (ICU) from aerosolization of fungi during building renovation. ${ }^{2}$ Primary invasive skin infections due to Aspergillus $\mathrm{Sp}$. have been reported in association with adhesive dressings for venous access devices which are contaminated with Aspergillus spores. ${ }^{4,5}$

In the present case patient admit that he was immunocompetent, and his HIV rapid test was negative. Numerous reports have described primary or secondary cutaneous aspergillosis in an array of non HIV-infected immunocompromised patients, including burn victims, neonates, individuals with cancer, and bone marrow and solid organ transplant recipients. In addition, otherwise healthy hosts can develop cutaneous aspergillosis in surgical wounds, by traumatic inoculation, or by exposure to high spore counts in occupations such as farming. ${ }^{6,7}$

The skin lesions of primary cutaneous aspergillosis occurs as a result of direct inoculation of Aspergillus spores at the site of injury. It may present most commonly on the extremities with several form. It may first appear as erythematous papules, then become pustule, and subsequently develop a central ulceration with an elevated border that is covered by a black eschar. Although this latter stage is characteristic of Aspergillus skin lesions, the appearance is not pathognomonic. 8 Primary cutaneous aspergillosis may also arising in a wound generally presents with significant fever, a change in the character of the wound surface, swelling, induration, and tenderness. ${ }^{2}$ Aspergillus species is a saprophytic mold, which is found in decaying organic matter. Infection occurs by inhalation of spores or by direct entry of organisms into body tissue through wound. ${ }^{5}$ This quite same with literature that patient chief complaint is itchy redness macule and pimples, and from examination there are erythematous macule unsharply marginated with papules but in this patient there are no complaint about fever, only felt itchy.

Presumptive diagnosis of primary cutaneous aspergillosis can be made immediately by examining the lesion with potassium hydroxide preparation of a biopsy specimen. Generally, however, the diagnosis of most primary and secondary Aspergillus infections requires biopsy of a skin lesion taken from both culture and histopathology. A skin biopsy specimen for a suspected fungal lesion should be taken from the center of the lesion and should reach the subcutaneous fat because Aspergillus tends to invade blood vessels of the dermis and subcutis. ${ }^{8}$ In this patient we done the potassium hydroxide with the result shows conidiophores, dichotomously branching and septate hyphae. The hyphae of Aspergillus have a relatively characteristic size (3-6 lm) and morphology (septate with progressive arboreal and dichotomous branching). Histological examination is necessary because it shows invasion of tissues and demonstrates the pathogenicity of the mycete; however, diagnosis of primary cutaneous aspergillosis must also be based on culture. ${ }^{4}$

These colonies were taken and cultured on SDA and incubated at $34^{\circ} \mathrm{C}$ in a room temperature colonies are granular, flat often with radial grooves, yellow at first but quickly becoming bright to dark yellow-green according to age were observed on the agar. Microscopically, the colonies showed the colonies with the conidial head are typically radiate, later splitting to form loose column, biseriate but having some head with phialides borne directly on the vesicle (unisareate), conidiophore stripes are hyaline and coarsely roughned often more noticeable near the vesicle, and the fungus was identified as Aspergillus flavus. Some literature said that the specimen should be minced and plated on medium specific for the recovery of yeast (e.g., bromcresol green), mold (e.g., potato dextrose agar), and dermatophytes (e.g., Mycobiotic) and should be held for 6 weeks. The remaining specimen should be used for the recovery of bacteria by plating homogenized specimen on blood agar for 48 hour and incubating the specimen in thioglycolate broth for 7 days. Fungal isolates from culture media are identified on the basis of colony morphology, color, and sporulation. The variations of colony morphology in Aspergillus are many, depending upon the species. Most species begin as white colonies, but rapidly develop colors of green, yellow, orange, black or brown. Colonies are fluffy to velvet- like and are mature in 3-5 days. Unfortunately, blood cultures have low sensitivity, even for Aspergillus endocarditis. Aspergillus species grow well on standard media and can be identified to species level in most laboratories, the aspirate was inoculated on SDA with and without gentamycin and incubated at $26^{\circ} \mathrm{C}$ until $37^{\circ} \mathrm{C} .1,9,10$

Culture confirmation, where possible, is important to differentiate aspergillosis from other filamentous fungal infections, such as fusariosis and scedosporiosis. Blood cultures are of limited utility, because the results are often not positive even in disseminated infection. ${ }^{11}$ Aspergillus $S p$. is the most ubiquitous fungi that exist in soil, water and decaying vegetations. Although there are over 350 species of Aspergillus, widely distributed in nature, only a few are 
pathogenic to man and often Aspergillus fumigatus and Aspergillus flavus are the offenders in systemic infections. Cutaneous aspergillosis is mostly caused by Aspergillus flavus and Aspergillus fumigatus and rarely by Aspergillus niger, Aspergillus terreus, Aspergillus ustus, and Aspergillus chevalieri. ${ }^{1,12}$

A skin biopsy specimen for a suspected fungal lesion should be taken from the center of the lesion and should reach the subcutaneous fat because Aspergillus tends to invade blood vessels of the dermis and subcutis. If a single biopsy specimen is taken, the biopsy specimen should be divided and one half should be sent in saline to the microbiology laboratory and the other half should be sent in formalin to the pathology laboratory. In the microbiology laboratory, fungal hyphal structures can be stained directly from tissue specimens. The specimen should be minced and plated on medium specific and should be held for 2-6 weeks. Fungal isolates from culture media are identified on the basis of colony morphology, color, and sporulation. In the pathology laboratory, histopathologic examination with routine stains, such as hematoxylin and eosin, variably demonstrates Aspergillus hyphae, sometimes staining the nucleus or cytoplasm of the fungus or revealing the cell wall by a negatively staining shadow. The cellular infiltrate of Aspergilluslesions is not distinct. Aspergillus hyphae should have acute-angle branching and frequent septations. ${ }^{8}$ Histopathologically by tissue sections, diagnosis can be made by demonstration of nonpigmented septated hyphae that branch at acute angles. Hematoxylin eosin staining of the skin biopsy specimen demonstrated dermal diffuse and nodular granulomatous infiltration with large amounts of lymphocytes, multinucleated giant cell, eosinophil, and neutrophil. ${ }^{12,13}$ But in this patients the biopsy is not performed because the patient refuses on her first visit because still felt pain in approximately in the lesions that also post operation wound, and on his last visit when the patient wants to do a biopsy, but the results potassium hydroxide obtained are already negative.

When aspergillosis is diagnosed, subsequent efforts should be directed at determining whether the patient has a primary infection or whether there is secondary dissemination from a primary focus such as the lung. The workup should begin with an assessment of risk factors (neutropenia, recent or concurrent presence of a central venous access catheter, the presence of adhesive or occlusive dressings, or other local skin injury). Special attention to pulmonary symptoms and/or signs may determine whether an evaluation for pulmonary aspergillosis is needed. If there are indications of pulmonary infection, a computed tomographic scan of the chest would be the best first diagnostic test. If that test is abnormal, evaluation by bronchoscopy should follow. The detection of antigen or antibody in serum has not been studied for cutaneous aspergillosis, and the sensitivities of these tests are anticipated to be sufficiently low that there will be no immediate role for their clinical application. ${ }^{14,15}$

Treatment approach to cutaneous aspergillosis generally depends on the underlying status of the patient. Cutaneous aspergillosis in burn victims occurs as primary disease, treated principally with a surgical approach that may involve amputation. Conversely, the approach in premature neonates with cutaneous aspergillosis, who do not tolerate skin surgery well, is antifungal chemotherapy without surgery. Cancer and bone marrow transplant recipients have received a variety of medical and surgical treatments that have included immunomodulating granulocyte transfusions in one case and skin grafting in other cases. With a combination of organism directed medical therapy and surgical excision, most HIV related cases of primary cutaneous aspergillosis lesions did not recur. The risk of dissemination with either tape associated or catheterrelated primary cutaneous aspergillosis cases among HIV infected patients appeared low but did occur in two of nine patients. As the presence of secondary cutaneous lesions reflects disseminated infection, therapy with systemic voriconazole was recommended as primary therapy. Surgical intervention, particularly for primary cutaneous infection, may be useful. ${ }^{16}$

Systemic antifungal therapy is the mainstay of therapy, and the results are generally good. Surgical excision may occasionally be necessary when the local infection cannot be controlled in the neutropenic setting. In catheter site infections, removal of the catheter in addition to systemic antifungal therapy is indicated. Burn wound aspergillosis and posttraumatic soft tissue infections are best managed by surgical debridement in addition to systemic therapy.

Itraconazole has been used for the treatment of cutaneous aspergillosis. Its mode of action is through the inhibition of the cytochrome P-450-dependent demethylation stage in the formation of ergosterol on the fungal cell membrane. Itraconazole is well absorbed orally, and because of its highly lipophilic character, it is accumulated in the tissue at a higher level than in the plasma. The bioavailability of the drug is increased if it is taken with a fatty meal, but can be decreased in patients taking drugs that impair gastric acidity, such as histamine-2 blockers and antacids. ${ }^{12,17}$ In vitro, it is fungistatic and effective against dermatophytes, yeast, molds, and dimorphic fungi. Itraconazole inhibits 14$\alpha$-demethylase, a microsomal cytochrome P450 enzyme, in the fungal membrane. 14- $\alpha$-Demethylase is 
necessary for the conversion of lanosterol to ergosterol, which is the principal structural component of the fungal cell membrane. Consequently, the accumulation of 14- $\alpha$-methylsterols leads to the impairment of membrane permeability and membrane-bound enzyme activity and to the arrest of fungal cell growth. ${ }^{18}$

The recommended dosage range of oral itraconazole in adults is $400 \mathrm{mg}$ /day (capsules) and 2.5 $\mathrm{mg} / \mathrm{kg}$ twice daily (solution). In pediatric patients aged 15 years, a dosage of oral itraconazole solution of 2.5 $\mathrm{mg} / \mathrm{kg}$ twice daily has been recommended [100]. The approved adult dosages of IV itraconazole are $200 \mathrm{mg}$ twice daily for 2 days, followed by $200 \mathrm{mg}$ once daily for a maximum of 12 days. Because of the erratic bioavailability of itraconazole, measurements of plasma concentrations of itraconazole by bioassay are recommended during oral therapy of invasive aspergillosis. ${ }^{7,17}$ Patients received itraconazole therapy for 4 weeks and the results obtained potassium hydroxide that is already out of the picture that fit the description Aspergillus Sp., but only found a lot of hyphae then treatment is continued for 2 weeks and giving excellent therapeutic response.

\section{REFERENCES}

1. Venugopal TV, Venugopal PV. Primary cutaneous aspergillosis from Tamilnadu diagnosed by fine needle aspiration cytology. ISHAM 2012; 221:103-6.

2. Tahir C, Garbati M, Nggada HA, Yawe EH, Abubakar AM. Case report primary cutaneous aspergillosis in an immunocompetent patient. J Surg Tech Case Report 2011; 3(2): 94-6.

3. Dal T, Tekin A, Tekin R, Deveci O, Firat U, Mete $\mathrm{M}$, et al. Case report soft tissue abscess caused by aspergillus fumigatus in an immunosuppressive patient. Eur J Gen Med 2013; 10(2): 118-22.

4. Uniyal V, Bhatt RP, Saxena S, Talwar A. Antifungal activity of essential oils and their volatile constituents against respiratory tract pathogens causing aspergilloma and aspergillosis by gaseous contact. IJANS 2012; 4(1): 65-70.

5. Saunte DML, Schaller M. Non-dermatophytes maoulds in dermatology. Mycoses. 2015;58 (4): 16

6. Chaturvedi R, Kolhe A, Pardeshi K, Naik L, Wanjare S. Primary cutaneous aspergillosis, mimicking malignancy, a rare presentation in an immunocompetent patient. Diagnostic
Cytopathology 2017; 1(1):1-4.

7. Nakashima K, Yamada N, Yoshida Y, Yamamoto O. Primary cutaneous aspergillosis. Acta Derm Venereol 2010; 10: 519-25.

8. Darr-Foit, S. Primary cutaneous aspergillosis-an uncommon opportunistic infection. Journal of the German Society of Dermatology. 2017;83941

9. Rocha PS, Pinto RGW, Rodrigues S, Rodrigues MJP, Sudhakaran AA. Cytodiagnosis of primary cutaneous aspergillosis in an immunocompetent host. J Cytol. 2016;33(1):59-60

10. Bernardeschi C, Foulet F, Ingen-Housz-Oro S. Cutaneous invasive aspergillosis: retrospective multicenter study of the french invasiveaspergillosis registry and literature review. Medicine (Baltimore) 2015; 94: 10-8.

11. Sciortino C. Atlas of Clinically Important Fungi. New Jersey: John Wiley and Sons. 2017. pp5968.

12. Tatara AM, Mikos AG, Kontoyiannis DP. Factors affecting patient outcome in primary cutaneous aspergillosis. MD Journal 2016; 9(26): 1-7.

13. Liu X, Yang J, Ma Weiyuan. Primary cutaneous aspergillosis caused by Aspergillus fumigatus in an immunocompetent patient: a case report. Medicine (Baltimore). 2017;96(48):8916

14. Tunccan OG, Aki SZ, Akyurek N, Sucak G, Senol E. Case report isolated cutaneous aspergillosis in an acute lymphoblastic leukemia patient after allogeneic stem cell transplantation. J Infect Dev Ctries 2011; 5(5): 406-9.

15. Georgiadou SP, Kontoyiannis DP. Concurrent lung infections in patients with hematological malignancies and invasive pulmonary aspergillosis: how firm is the aspergillus diagnosis. J Infect 2012; 65: 262-8.

16. Lohana P, Hogg FJ. Vacuum assisted closure and primary cutaneous aspergillosis in a burn a management dilemma. Ann Burns Fire Disasters 2010; 23: 48-50.

17. Sigurgeirsson B, Hay RJ. Antibiotics and antifungal therapy in dermatology: The antifungal drugs used in skin disease. Springer International Publishing 2016; 1(1): 147-148.

18. Ehst BD, Blauvelt A. Skin Disease in Acute and Chronic Immunosupression. Fitzpatrick's Dermatology in General Medicine $8^{\text {th }}$ edition. New York: McGrawhill. 2012(29): pp330-344 